\title{
Recent results on bottomonium spectroscopy from BABAR
}

Bill Gary for the BABAR Collaboration

Department of Physics and Astronomy, University of California, Riverside, CA 92521, USA

DOI: http://dx.doi.org/10.5689/UA-PROC-2010-09/08

\begin{abstract}
Recent results from the BABAR experiment on bottomonium spectroscopy are presented. The results include the first observation of the $\Upsilon\left(1^{3} D_{J}\right)$ in a hadronic decay channel, a study of the $\eta_{b}$ using photon conversions, and searches for the as-yet-unseen $h_{b}(1 P)$.
\end{abstract}

\section{Introduction}

The BABAR experiment operated at SLAC from 1999 to 2008. It collected data in the vicinity of the $\Upsilon$ system. The primary data sample (around $430 \mathrm{pb}^{-1}$ ) was collected on the $\Upsilon(4 S$ ) resonance and was used for studies of $C P$ violation in $B$ meson decays, for CKM physics, for charm meson physics, for $\tau$ lepton physics, and other studies. In the final two months of operation, the energy was lowered so that large samples on the $\Upsilon(3 S)$ and $\Upsilon(2 S)$ resonances could be collected. In particular, the world's largest sample of $\Upsilon(3 S)$ events (by more than a factor of 10) was collected. $30 \mathrm{fb}^{-1}$ were collected at the $\Upsilon(3 S)$, corresponding to 120 million $\Upsilon(3 S)$ decays. The corresponding figures for the $\Upsilon(2 S)$ are $14 \mathrm{fb}^{-1}$ and 100 million.

The spectrum of states below the open-flavor threshold is much richer for bottomonium than for charmonium, i.e., a lot more states are expected. The masses and branching fractions of these states provide important tests for QCD potential models and lattice QCD. Hadronic transitions between bottomonium states probe non-perturbative QCD.

Bottomonium states with orbital angular momentum $L=0$ or 1 and total quark spin 1 , namely the $\Upsilon(n S)$ and $\chi_{b J}(n P)$ states, were observed in the 1970s and 1980s. There was then a long period during which no additional bottomonium states were discovered. This ended in 2004, when CLEO observed the $\Upsilon\left(1^{3} D_{2}\right)$ [1]. Subsequently, in 2008, BABAR observed the $\eta_{b}$ [2]. Unlike charmonium, many bottomonium states below the open-flavor threshold have not yet been seen.

Three topics in bottomonium spectroscopy, based on the BABAR $\Upsilon(3 S)$ sample, are presented here: a search for the $\eta_{b}$ (the singlet $L=0$ state of bottomonium) using $\gamma \rightarrow e^{+} e^{-}$conversions, a search for the $h_{b}(1 P)$ (singlet $L=1$ state), which has not yet been seen, and the first observation of hadronic decays of the $\Upsilon\left(1^{3} D_{2}\right)$ (triplet $L=2$ ) state. Besides the $\Upsilon(3 S)$ sample, the $\eta_{b}$ study makes use of the $\Upsilon(2 S)$ sample. All results are preliminary. 


\section{Search for the $\eta_{b}$ in photon conversions}

The $\eta_{b}$ was discovered [2] by BABAR in the recoil $\gamma$ spectrum of $\Upsilon(3 S) \rightarrow \gamma \eta_{b}$ decays. It was subsequently seen by BABAR in $\Upsilon(2 S)$ decays [3] and confirmed by CLEO [4]. In the new study, a search for the $\eta_{b}$ is made in the $\Upsilon(3 S) \rightarrow \gamma \eta_{b}$ and $\Upsilon(2 S) \rightarrow \gamma \eta_{b}$ channels using photon conversions, $\gamma \rightarrow e^{+} e^{-}$. Converted photons have 5 times better energy resolution than the calorimeter photons used in the previous $\eta_{b}$ studies $(5 \mathrm{MeV}$, rather than $25 \mathrm{MeV})$. Thus, the study based on converted photons can potentially help to improve the $\eta_{b}$ mass measurement. The efficiency for converted photons is is lower than for calorimeter photons. Nonetheless an $\eta_{b}$ signal on the order of 3 standard deviations is expected.

There are five monochromatic photons expected in the vicinity of the $\eta_{b}$ signal photons: one from the signal photon itself, one from initial-state photon radiation (ISR) in $e^{+} e^{-} \rightarrow$ $g_{I S R} \Upsilon(1 S)$, and three from $\chi_{b J} \rightarrow \gamma \Upsilon(1 S)$ transitions $(J=0,1,2)$. After identifying $\gamma \rightarrow e^{+} e^{-}$ conversions with a conversion algorithm and applying vetoes against $\gamma$ candidates from $\pi^{0}$ decay and other cuts, we perform a $\chi^{2}$ fit to the $\gamma$ recoil spectrum accounting for the five "peaking" components and a combinatoric background. The fit is made simultaneously to the $\Upsilon(3 S)$ and $\Upsilon(2 S)$ data with the $\eta_{b}$ mass a fitted parameter.
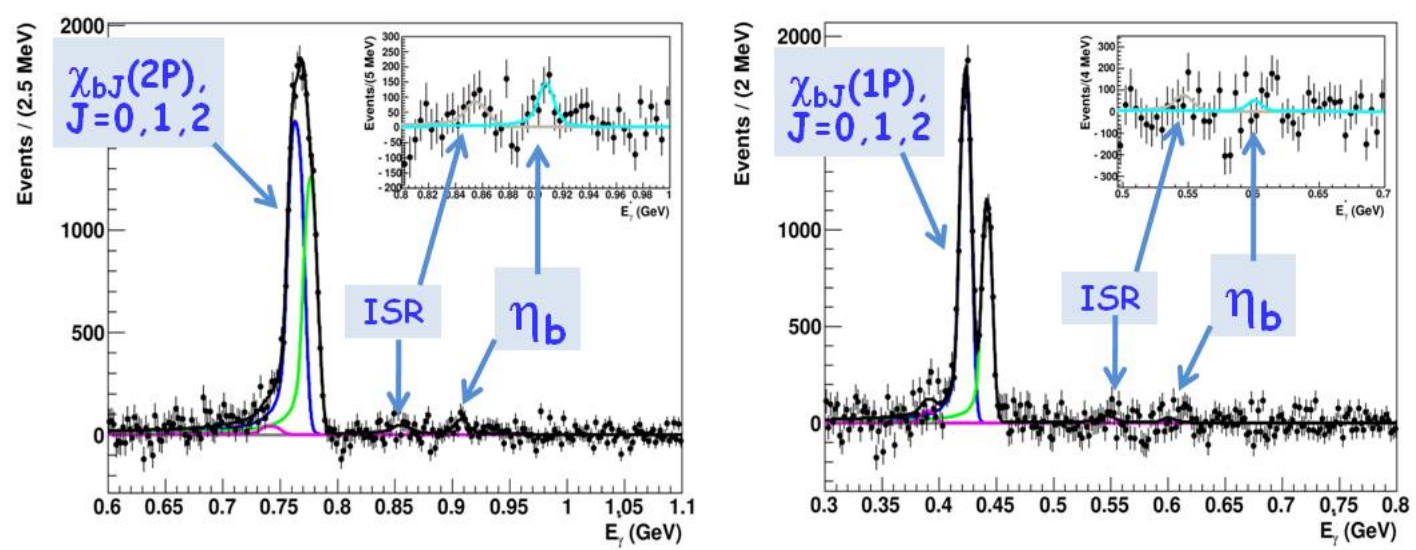

Figure 1: The $\gamma$ recoil spectrum after subtraction of continuum background for the $\Upsilon(3 S)$ (left) and $\Upsilon(2 S)$ (right) data.

The results of the fit are shown in Fig. 1. The results are shown after subtraction of the continuum background term. The prominent peaks are from the $\chi_{b J} \rightarrow \gamma \Upsilon(1 S)$ transitions. The ISR and $\eta_{b}$ contributions are indicated by the arrows. $773 \pm 220 \eta_{b}$ events are observed in the $\Upsilon(3 S)$ decays (770 are expected), compared to $289 \pm 281$ in the $\Upsilon(2 S)$ decays (390 expected). The combined $\eta_{b}$ significance is 2.6 standard deviations including systematic uncertainties. Since the significance of the $\eta_{b}$ observation is less than 3 standard deviations, it is difficult to make a conclusive statement about the fitted mass value: this is still under investigation.

As a side-benefit of this analysis, we obtain the world's best measurements for the separated $\Upsilon(n S) \rightarrow \gamma \chi_{b J}[(n-1) P] \rightarrow \gamma \gamma \Upsilon(1 S)$ branching fractions $(n=2,3)$. For the $J=1$ and 2 states, we improve the PDG averages by factors of about $2.5-3$ for the $\chi_{b J}(2 P)$ transitions and $3-4$ for the $\chi_{b J}(1 P)$ transitions. 


\section{Search for the $h_{b}(1 P)$ state}

The $h_{b}(1 P)$ is a $P$-wave singlet state, as mentioned above. It is the hyperfine-splitting partner of the $\chi_{b J}(1 P)$ states. This hyperfine splitting is expected to be very small, leading to a firm expectation that the $h_{b}(1 P)$ mass is close to $9900 \mathrm{MeV} / c^{2}$. Decays of the $\Upsilon(3 S)$ to $\gamma h_{b}(1 P)$, analogous to those discussed above the for $\eta_{b}$, are forbidden by charge-conjugation symmetry. The favored production mechanisms are hadronic: $\Upsilon(3 S) \rightarrow \pi^{0} h_{b}(1 P)$ and $\Upsilon(3 S) \rightarrow$ $\pi^{+} \pi^{-} h_{b}(1 P)$. Voloshin [5] predicts that the former decay dominates over the latter, while Kuang et al. [6] predict the opposite. However, there is much uncertainty in the predictions. The $h_{b}(1 P)$ is expected to decay around $40 \%$ of the time to $\gamma \eta_{b}$. The monochromatic photon in this decay, of energy $490 \mathrm{MeV}$ (resolution $25 \mathrm{MeV}$ ), can be used to help isolate an $h_{b}(1 P)$ signal.
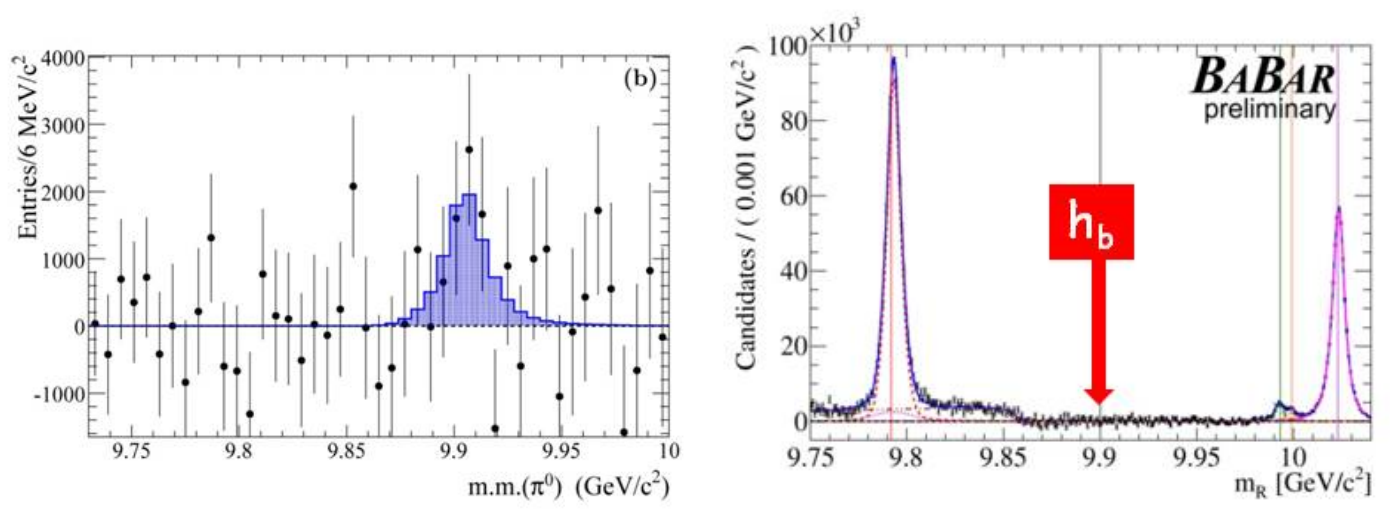

Figure 2: (left) The recoil spectrum against the $\pi^{0}$ in $\Upsilon(3 S) \rightarrow \pi^{0}+X$ events. (right) The recoil spectrum against the $\pi^{+} \pi^{-}$pair in $\Upsilon(3 S) \rightarrow \pi^{+} \pi^{-}+X$ events.

We search for the $h_{b}(1 P)$ in the $\Upsilon(3 S) \rightarrow \pi^{0} h_{b}(1 P)$ channel. We require the presence of a photon consistent with the $h_{b}(1 P) \rightarrow \gamma \eta_{b}$ decay. $\pi^{0} \rightarrow \gamma \gamma$ candidates are reconstructed, and cuts are applied to reduce background. The overall reconstruction efficiency is $22 \%$. We perform a $\chi^{2}$ fit to the recoil mass against the $\pi^{0}$, accounting for a smooth combinatorial background and a $h_{b}(1 P)$ signal peak. The $h_{b}(1 P)$ mass and yield are determined in the fit. The results after subtraction of the combinatoric background are shown in the left-hand plot of Fig. 2. The fitted mass of $9903 \pm 4 \mathrm{MeV} / c^{2}$ agrees with the expectation of $9900 \mathrm{MeV} / c^{2}$. The statistical significance is 2.7 standard deviations including systematic uncertainties. We are currently refining the analysis.

We also search for the $h_{b}(1 P)$ in the $\Upsilon(3 S) \rightarrow \pi^{+} \pi^{-} h_{b}(1 P)$ channel. We select $\pi^{+} \pi^{-}$ pairs that originate at the interaction point. After removing obvious $K_{S}^{0}$ candidates, we plot the recoil mass against the $\pi^{+} \pi^{-}$pair. We do not require a monochromatic photon from $h_{b}(1 P) \rightarrow \gamma \eta_{b}$ decays for the $\Upsilon(3 S) \rightarrow \pi^{+} \pi^{-} h_{b}(1 P)$ channel because this channel has less combinatoric background than the $\Upsilon(3 S) \rightarrow \pi^{0} h_{b}(1 P)$ channel and this requirement is not necessary. The reconstruction efficiency is $42 \%$. The results after subtraction of the combinatoric background are shown by the right-hand plot in Fig. 2. The large peak at around $9.80 \mathrm{GeV} / c^{2}$ is from $\Upsilon(2 S) \rightarrow \pi^{+} \pi^{-} \Upsilon(1 S)$ transitions and that at around $10.02 \mathrm{GeV} / c^{2}$ from $\Upsilon(3 S) \rightarrow \pi^{+} \pi^{-} \Upsilon(2 S)$ transitions. The less prominent structure just below $10.0 \mathrm{GeV} / c^{2}$ is from 
$\chi_{b J}(2 P) \rightarrow \pi^{+} \pi^{-} \chi_{b J}(1 P)$ transitions. At the value of $9.900 \mathrm{GeV} / c^{2}$ expected for the $h_{b}(1 P)$ mass, there is no indication of a signal. Therefore we have no evidence for the $h_{b}(1 P)$ in this channel.

\section{Hadronic decays of the $\Upsilon\left(1^{3} D_{2}\right)$}

The $\Upsilon\left(1^{3} D_{2}\right)$ state was first observed in 2004, by CLEO, as mentioned in the introduction. It was observed in the radiative $\Upsilon\left(1^{3} D_{2}\right) \rightarrow \gamma \gamma \Upsilon(1 S)$ decay channel. The $\Upsilon\left(1^{3} D_{J}\right)$ is a triplet $D$-wave state. The signal seen by CLEO was consistent with a single state, interpreted to be the $J=2$ member of the triplet.

In our study, we search for $\Upsilon\left(1^{3} D_{J}\right)$ states produced in the decay chain $\Upsilon(3 S) \rightarrow \gamma \gamma \Upsilon\left(1^{3} D_{J}\right) \rightarrow$ $\gamma \gamma \pi^{+} \pi^{-} \Upsilon(1 S)$, with $\Upsilon(1 S) \rightarrow \ell^{+} \ell^{-}(\ell=e, \mu)$. The hadronic decay channel $\Upsilon\left(1^{3} D_{J}\right) \rightarrow$ $\pi^{+} \pi^{-} \Upsilon(1 S)$ has been of interest for decades $[7,8,9,10]$. Predictions for its branching fraction vary widely $[8,9,10]$. It provides better mass resolution than the radiative channel studied by CLEO and allows the $L, J$, and $P$ quantum numbers, for which there is currently no experimental information, to be tested, through measurement of the angular distributions of the $\pi^{ \pm}$ and $\ell^{ \pm}$.

To search for $\Upsilon(3 S) \rightarrow \gamma \gamma \Upsilon\left(1^{3} D_{J}\right) \rightarrow \gamma \gamma \pi^{+} \pi^{-} \Upsilon(1 S)$ decays, we require exactly four charged tracks in an event, two of which are identified as pions with opposite charge and the other two as either an $e^{+} e^{-}$or $\mu^{+} \mu^{-}$pair. The $\Upsilon(1 S)$ candidate is selected by requiring $-0.35<$ $m_{e^{+} e^{-}}-m_{\Upsilon(1 S)}<0.2 \mathrm{GeV} / c^{2}$ or $\left|m_{\mu^{+} \mu^{-}}-m_{\Upsilon(1 S)}\right|<0.2 \mathrm{GeV} / \mathrm{c}^{2}$. The pion pair is combined with the $\Upsilon(1 S)$ candidate to form a $\Upsilon\left(1^{3} D_{J}\right)$ candidate. Two photons consistent with the expected decay chain are required. The $\Upsilon\left(1^{3} D_{J}\right)$ candidate is combined with the two photons to form a $\Upsilon(3 S)$ candidate. The CM momentum and energy of the $\Upsilon(3 S)$ are required to be consistent with zero and $\Upsilon(3 S)$ mass, respectively. The overall selection efficiency is around $26 \%$.
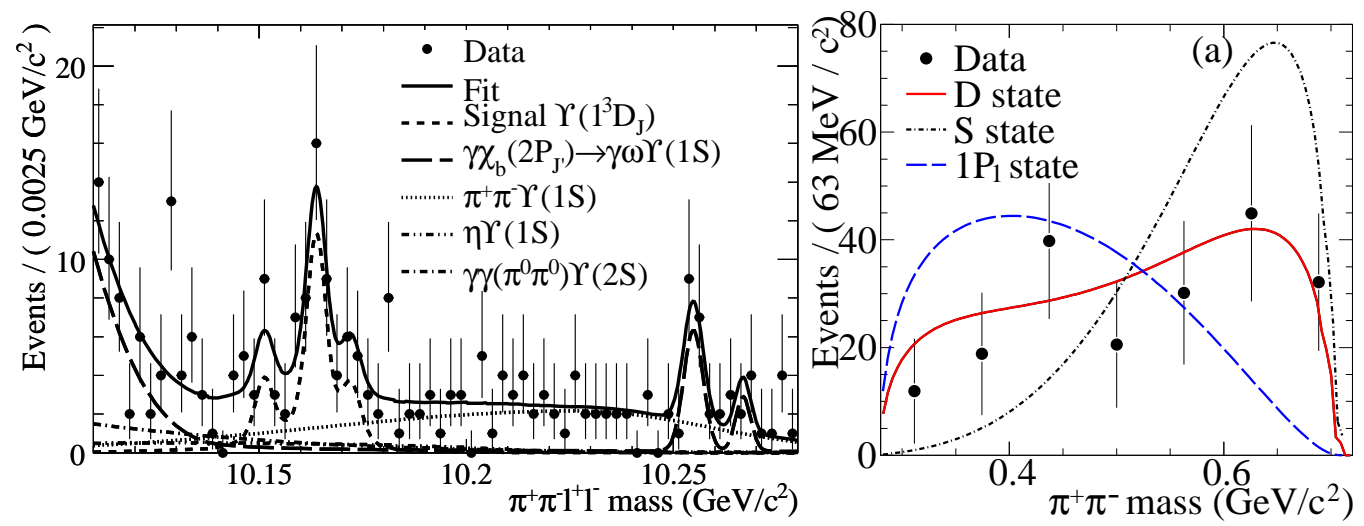

Figure 3: (left) The recoil spectrum against the $\pi^{0}$ in $\Upsilon(3 S) \rightarrow \pi^{0}+X$ events. (right) The recoil spectrum against the $\pi^{+} \pi^{-}$pair in $\Upsilon(3 S) \rightarrow \pi^{+} \pi^{-}+X$ events.

We apply an unbinned maximum likelihood fit to the selected events. The fit has a component for each of the three $\Upsilon\left(1^{3} D_{J}\right)$ signal states as well as several background categories. The result of the fit is shown in the left-hand portion of Fig. 3. We obtain a yield of 
$33.9_{-7.5}^{+8.2} \Upsilon\left(1^{3} D_{2}\right)$ events, corresponding to a significance of 5.8 standard deviations including systematic uncertainties. The significances of the $\Upsilon\left(1^{3} D_{1}\right)$ and $\Upsilon\left(1^{3} D_{3}\right)$ states are less than 2 standard deviations after systematics are included. We determine the $\Upsilon\left(1^{3} D_{2}\right)$ mass to be $10164.5 \pm 0.8 \pm 0.5 \mathrm{MeV} / c^{2}$, which improves the CLEO result by almost a factor of two. We determine the $\Upsilon\left(1^{3} D_{J}\right) \rightarrow \pi^{+} \pi^{-} \Upsilon(1 S)$ branching fraction to be $0.66_{-0.14}^{+0.15} \pm 0.06 \%$, which lies between the predictions of about $0.2 \%$ from Ref. [9] and $2 \%$ from Ref. [10].

The right-hand portion of Fig. 3 shows the $\pi^{+} \pi^{-}$mass distribution for events in the $\Upsilon\left(1^{3} D_{J}\right)$ signal region $10.140<m_{\pi^{+} \pi^{-} \ell^{+} \ell^{-}}<10.178 \mathrm{GeV} / c^{2}$. Shown in comparison are the expectations for the decay of a $D, S$, or ${ }^{1} P_{1}$ bottomonium state to $\pi^{+} \pi^{-} \Upsilon(1 S)$. The resulting $\chi^{2}$ probabilities of $84.6 \%, 3.1 \%$, and $0.3 \%$, respectively, strongly favor the $D$ state. This confirms the orbital angular momentum quantum number of the observed state. Similarly, we examine the distribution of the angle $\chi$ between the $\ell^{+} \ell^{-}$and $\pi^{+} \pi^{-}$planes in the $\Upsilon\left(1^{3} D_{2}\right)$ rest frame and find consistency with the expected assignments $J=2$ and $P=-1$ (total spin and parity).

\section{Summary}

In summary, BABAR collected the world's largest sample of $\Upsilon(3 S)$ events in 2008 , during the last two months the detector was in operation. A large sample of $\Upsilon(2 S)$ events was collected in addition. Three studies of bottomonium spectroscopy based on these data are presented here. Using converted photons in $\Upsilon(3 S)$ and $\Upsilon(2 S)$ decays to $\gamma \eta_{b}$, an $\eta_{b}$ signal with a significance of around 2.6 standard deviations (with systematics) is observed. This measurement may allow improvements in the $\eta_{b}$ mass determination. We improve the world measurements of the $\chi_{b J}(2 P) \rightarrow \gamma \Upsilon(1 S)$ and $\chi_{b J}(1 P) \rightarrow \gamma \Upsilon(1 S)$ branching fractions (separated by $J$ ) by factors of 3 to 4 . We find evidence for the as-yet-unseen $h_{b}(1 P)$ state at the level of about 2.7 standard deviations (including systematics) in the $\Upsilon(3 S) \rightarrow \pi^{0} h_{b}(1 P)$ channel. We have made the first observation of hadronic decays of the $\Upsilon\left(1^{3} D_{2}\right)$ state. The significance is 5.8 standard deviations including systematics. We improve the mass measurement of the $\Upsilon\left(1^{3} D_{2}\right)$ by a factor of two and provide the first tests of its quantum numbers.

\section{References}

[1] G. Bonvicini et al. (CLEO Collaboration), Phys. Rev. D 70, 032001 (2004).

[2] B. Aubert et al. (BABAR Collaboration), Phys. Rev. Lett. 101 (2008) 071801.

[3] B. Aubert et al. (BABAR Collaboration), Phys. Rev. Lett. 103 (2009) 161801.

[4] G. Bonvicini et al. (CLEO Collaboration), Phys. Rev. D 81 (2010) 031104.

[5] M.B. Voloshin, Sov. J. Nucl. Phys. 43 (1986) 1011.

[6] Y.-P. Kuang, S.F. Tuan, and T.-M. Yan, Phys. Rev. D 37 (1988) 1210.

[7] W. Kwong and J. L. Rosner, Phys. Rev. D 38, 279 (1988).

[8] Y.-P. Kuang and T.-M. Yan, Phys. Rev. D 24, 2874 (1981).

[9] P. Moxhay, Phys. Rev. D 37, 2557 (1988).

[10] P. Ko, Phys. Rev. D 47, 208 (1993). 